\title{
Treatment of central sleep apnoea in congestive heart failure with nasal ventilation
}

\author{
G N Willson, I Wilcox, A J Piper, W E Flynn, R R Grunstein, C E Sullivan
}

Cheyne-Stokes respiration (CSR) is common in patients with congestive heart failure $(\mathrm{CHF}){ }^{1}$ This characteristic crescendo-decrescendo pattern of breathing is often seen during sleep in patients with $\mathrm{CHF}$ and is a form of central sleep apnoea. ${ }^{2}$ Disordered nocturnal breathing leads to oxygen desaturation, poor sleep quality, and altered sleep architecture. ${ }^{3}$ These features may lead to complaints of daytime somnolence, fatigue, insomnia, and many of the symptoms typical of sleep disordered breathing.

It has been proposed that CSR during sleep in patients with heart failure is an indicator of a poor prognosis. ${ }^{45}$ The likely adverse effects of CSR on daytime performance and myocardial function have resulted in the introduction and evaluation of a range of treatments aimed at reducing CSR. These include low flow oxygen, ${ }^{6}$ theophylline, ${ }^{7}$ and nasal continuous positive airways pressure (nCPAP). ${ }^{8}$ In one controlled study nCPAP was shown to improve both sleep quality and daytime myocardial function. ${ }^{8}$ Other groups have found nCPAP to be ineffective in controlling CSR. ${ }^{9-11}$

Nocturnal nasal intermittent positive pressure ventilation (nIPPV) is the accepted treatment for patients with chronic respiratory failure due to hypoventilation during sleep. ${ }^{12}{ }^{13} \mathrm{We}$ hypothesised that nIPPV may also effectively treat central sleep apnoea in heart failure by controlling ventilation and carbon dioxide in patients whose disordered breathing has been linked to fluctuating carbon dioxide levels, relative hyperventilation, and hypocapnia. ${ }^{14}$ Nasal IPPV was administered in an attempt to mechanically ventilate the patient through the apnoeic portion of the Cheyne-Stokes cycle, thereby preventing fluctuations in carbon dioxide levels and consequently abolishing CSR.

This study describes the use of nocturnal nIPPV in a group of patients with heart failure and Cheyne-Stokes respiration during sleep. The effects of this therapy on respiratory disturbance and sleep quality following an in-hospital acclimatisation period are reported. We also report our experience with long term domiciliary therapy and its effects on daytime left ventricular function in patients who remained compliant with treatment.

\section{Methods}

PATIENT SELECTION

All patients referred to our centre in the period October 1993 to January 1996 were assessed for the study. Patients with moderate to severe stable chronic left ventricular heart failure were included. All subjects had documented severe repetitive central sleep apnoea (respiratory disturbance index, RDI $>30 / \mathrm{h}$ ) and minimal obstructive apnoea $(<10 \%$ of respiratory events). Patients were excluded if they had been admitted to hospital or changed their medication within one month of the study or if they were unable to tolerate a nasal mask (one patient). They were also excluded if there was a history of completed stroke or significant pulmonary disease. All patients consented to participate in the study and the protocol was approved by the ethics committee of our institution.

All patients were examined by a cardiologist to confirm both historical and clinical evidence of chronic heart failure. Subjects had measurements of anthropometric variables (height/ weight), awake arterial blood gas tensions, and left ventricular ejection fraction (LVEF) using radionuclide gated blood pool scanning with technetium-99 pertechnetate. The radionuclide studies were performed using the standard clinical protocol and reported by a physician who was blinded to the treatment status of the patient and the likely effects of nIPPV.

The subjects continued to take their normal cardiac medications throughout the period of the study. These medications were converting enzyme inhibitor (10 patients), diuretic (10 patients), digoxin (seven patients), amiodarone (two patients), long acting nitrates (three patients), calcium antagonist (one patient). No patient who took part in the analysis of sleep was taking any hypnotic or antidepressant medication.

SLEEP STUDIES

All patients had overnight full sleep studies whilst breathing room air. Studies were conducted between 21.00 hours and 07.30 hours. Sleep state was monitored using standard techniques. Breathing variables which were monitored included chest wall and abdominal motion, diaphragm electromyogram (EMGd), arterial oxyhaemoglobin saturation $\left(\mathrm{SaO}_{2}\right)$, transcutaneous carbon dioxide pressure $\left(\mathrm{PtcCO}_{2}\right)$, and nasal airflow. Electrocardiograms were measured continuously in all patients. All variables were recorded on a 16 channel polygraph.

Sleep stages were scored according to standard criteria and expressed as a percentage of total sleep time (TST). ${ }^{16}$ Sleep efficiency was defined as the percentage of the total recording time which was scored as sleep. Arousal was defined as an awakening for longer than three seconds. ${ }^{17}$

Apnoeas and hypopnoeas were scored according to standard criteria as previously described. ${ }^{18}$ Obstructive events whilst receiving nIPPV were scored in the presence of desaturation and
Department of Medicine DO6, University of Sydney, NSW 2006, Australia. 
arousal preceded by a transient period of increased peak inspiratory airway pressures.

INITIATION OF NASAL IPPV

Following the confirmation of the diagnosis of CSR in sleep, 11 patients were acclimatised to nasal ventilation. All patients used commercially available masks and head straps (Sullivan ${ }^{\mathrm{TM}}$, ResMed Inc, Sydney, Australia). A volume preset ventilator (PV 201, Breas, Goteborg, Sweden) was used to deliver room air whilst allowing the setting of tidal volume, breath rate, and inspiratory time. The aim of the titration protocol was to control CSR by providing mechanical ventilation. Breath rate and inspiratory time were set in such a way as to match the patient's awake breathing pattern, while tidal volume was set so that $\mathrm{PtcCO}_{2}$ was maintained at or slightly below the patient's awake level $(0.1-0.4 \mathrm{kPa})$. All patients needed a chin strap to prevent or minimise mouth leaks. Positive end expiratory pressure (PEEP) was applied to the expiratory valve if upper airway obstruction was present. Nasal ventilation was commenced on an inpatient basis, with the patient acclimatising to the device during daytime practice sessions and then gradually increasing the hours the machine was used at night. Over the first three days all patients were monitored continuously during sleep with both $\mathrm{SaO}_{2}$ and $\mathrm{PtcCO}_{2}$ to ensure the safety and efficacy of ventilation. Once acclimatised to the device, a repeat full sleep study on nIPPV was performed to ensure the adequacy of ventilation and to gauge the effect of treatment on both apnoea and sleep quality.

Seven patients underwent a third full sleep study off nIPPV following a period of domiciliary therapy. The purpose of this study was to assess the effect of nIPPV withdrawal on sleep disordered breathing.

DOMICILIARY THERAPY

All 11 patients were commenced on a home trial of nIPPV. The aim of this trial was to use nIPPV for at least a three month period and then to re-evaluate both subjective and objective responses to treatment. Repeat measures of LVEF were made in the seven patients who continued on treatment for at least one month. The most recent measure of cardiac function whilst on nIPPV was compared with baseline.
STATISTICAL ANALYSIS

All values are shown as mean (SD) unless stated otherwise. In the seven patients who had all three sleep studies we tested for an effect of treatment on respiratory disturbance, sleep fragmentation, and sleep architecture using two way repeated measures ANOVA followed by planned linear comparisons of ventilation night versus the mean of the two diagnostic nights and the difference between the two diagnostic nights. The changes in sleep variables from diagnostic to nIPPV nights and changes in LVEF were compared using paired Student's $t$ tests.

\section{Results}

PATIENTS

Eleven men with CHF and CSR during sleep were commenced on nIPPV. All patients continued with a domiciliary trial of the device. Ten patients were included in the analysis of nIPPV on sleep, one patient who received a short period of nCPAP during his diagnostic study being excluded from this part of the analysis. Seven patients underwent a repeat full sleep study off nIPPV. This repeat study was performed after a median (interquartile range) of 36 (28-78) nights on nIPPV.

Patients were referred from a variety of sources. Seven patients had undergone previous sleep studies with four having failed a treatment trial with nCPAP due to either poor compliance or inadequate therapeutic response. Referrals from both respiratory physicians and cardiologists directly to our sleep unit accounted for the remaining four patients. All subjects had symptoms of sleep apnoea syndrome with either a history of excessive daytime somnolence or witnessed apnoeas.

The patients' baseline clinical characteristics are shown in table 1 including anthropometric data, New York Heart Association (NYHA) functional class, LVEF, body mass index (BMI), and arterial blood gas tensions. The mean (SD) age of the patients was 70 (11) years (range 47-83). Patients were mostly nonobese with a mean BMI of $26.7(4.6) \mathrm{kg} / \mathrm{m}^{2}$, the highest being $32.8 \mathrm{~kg} / \mathrm{m}^{2}$. Left ventricular function was predominantly at least moderately impaired with an LVEF of 23 (9)\% (range $11-40 \%)$. The group as a whole had $\mathrm{PaCO}_{2}$

Table 1 Patient characteristics

\begin{tabular}{|c|c|c|c|c|c|c|c|c|c|}
\hline \multirow[b]{2}{*}{ Patient } & \multirow[b]{2}{*}{$\begin{array}{l}\text { Age } \\
\text { (years) }\end{array}$} & \multirow[b]{2}{*}{ Aetiology } & \multirow[b]{2}{*}{$\begin{array}{l}\text { LVEF } \\
(\%)\end{array}$} & \multirow[b]{2}{*}{ NYHA } & \multirow[b]{2}{*}{$\begin{array}{l}B M I \\
\left(\mathrm{~kg} / \mathrm{m}^{2}\right)\end{array}$} & \multicolumn{4}{|c|}{ Arterial blood gas tensions } \\
\hline & & & & & & $p H$ & $\begin{array}{l}\mathrm{PaO}_{2} \\
(\mathrm{kPa})\end{array}$ & $\begin{array}{l}\mathrm{PaCO}_{2} \\
(\mathrm{kPa})\end{array}$ & $\begin{array}{l}\mathrm{SaO}_{2} \\
(\%)\end{array}$ \\
\hline 1 & 47 & DCM & 22 & 3 & 32.8 & 7.31 & 14.5 & 4.7 & 98 \\
\hline 2 & 83 & CAD & 28 & 3 & 23 & 7.41 & 13.2 & 4.9 & 97 \\
\hline 3 & 54 & CAD & 16 & 3 & 30 & 7.42 & 11.7 & 4.7 & 98 \\
\hline 4 & 77 & CAD & 40 & 2 & 25.6 & 7.43 & 12.9 & 5.1 & 98 \\
\hline 5 & 70 & CAD & 11 & 3 & 18.1 & 7.48 & 13.7 & 4 & 98 \\
\hline 6 & 69 & CAD & 27 & 1 & 28.9 & 7.38 & 12.4 & 5.6 & 97 \\
\hline 7 & 72 & CAD & 25 & 2 & 26.1 & 7.39 & 12.9 & 5.3 & 97 \\
\hline 8 & 66 & CAD & 24 & 3 & 30.2 & 7.43 & 10.5 & 5.3 & 96 \\
\hline 9 & 58 & $\mathrm{CAD}$ & 13 & 3 & 27.2 & 7.43 & 8.1 & 4.5 & 92 \\
\hline 10 & 73 & DCM & 13 & 4 & 20.5 & 7.41 & 9.2 & 5.3 & 94 \\
\hline 11 & 60 & CAD & 28 & 4 & 31.4 & 7.41 & 9.6 & 5.1 & 95 \\
\hline Mean (SD) & $70(11)$ & & $23(9)$ & 2.8 & $26.7(4.6)$ & $7.40(0.04)$ & $11.7(2.1)$ & $5.0(0.5)$ & $96(2)$ \\
\hline
\end{tabular}

NYHA = New York Heart Association functional class; CAD = coronary artery disease DCM = dilated cardiomyopathy; $\mathrm{BMI}=$ body mass index; LVEF = left ventricular ejection fraction. 
values at the lower end of the normal range with a mean value of $5.0(0.5) \mathrm{kPa}$.

SLEEP STUDIES

The diagnostic sleep study demonstrated that all patients had significant central sleep apnoea with a mean RDI of 54 (14) events/h and a minimum $\mathrm{SaO}_{2}$ of 77 (5)\%. Apnoeic events were predominantly central in nature with a Cheyne-Stokes pattern. Seven patients had some degree of partial upper airway obstruction which was evidenced by either snoring ( $\mathrm{n}$ $=2$ ) or partial obstruction ( $n=7)$, identified by airflow notching during the period of hyperpnoea following central apnoea. The mean interval between the commencement of ventilation and the repeat sleep study was 4.2 days (range 1-7).

The use of nIPPV during sleep abolished CSR in all patients with a reduction in the RDI to 5 (6) events/h ( $\mathrm{p}<0.001$; fig 1 ). Residual events were due to upper airway obstruction. There was a marked reduction in the total number of arousals from 48 (14) to 14 (6) events/h ( $p<0.001)$, whilst arousals due to non-respiratory causes increased when using nIPPV (8 (6) versus 13 (5) events/h, p <0.01; fig 2). Patient 11 had a similar response to the group as a whole with a marked reduction in RDI from 54 (time on CPAP excluded) to 4 events/h.

The TST and sleep efficiency were unaffected by the introduction of nIPPV. Sleep architecture was modified by nIPPV with a significant reduction in stage $1 / 2$ sleep (82 (9)\% during control versus 64 (16)\% during nIPPV, $\mathrm{p}<0.01)$ and an increase in slow wave sleep (6 (6)\% during control versus 19 (17)\% during nIPPV, $\mathrm{p}<0.05$; fig 3). There was a small but non-significant increase in REM sleep from 13 (6) $\%$ during the control period to 17 (6)\% during nIPPV ( $\mathrm{p}=0.08$; fig 3 ).

Table 2 summarises the sleep study data for the seven patients who underwent a repeat sleep study off nIPPV. No significant difference was noted in any of the sleep related variables between the initial study and the study off nIPPV.

During the night of treatment with nIPPV the $\mathrm{PtcCO}_{2}$ (mean of the first 10 minutes of stable stage $1 / 2$ sleep) fell slightly from 5.78 $(0.82)$ to $5.48(0.51) \mathrm{kPa}$ in nine patients (data

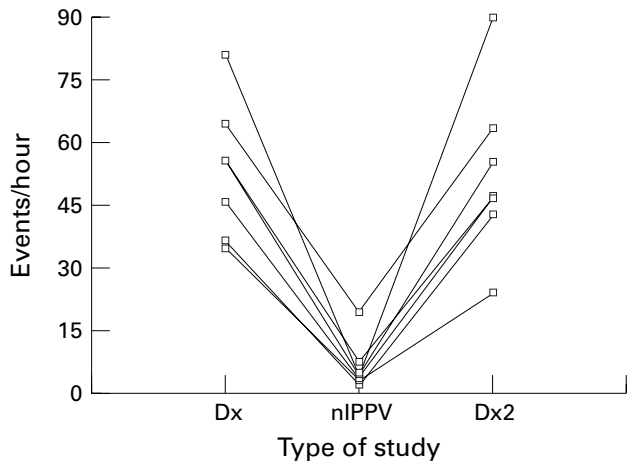

Figure 1 Respiratory disturbance index. Dx = baseline diagnostic; $n I P P V=$ on $n I P P V ; D \times 2=$ repeat study off nIPPV.
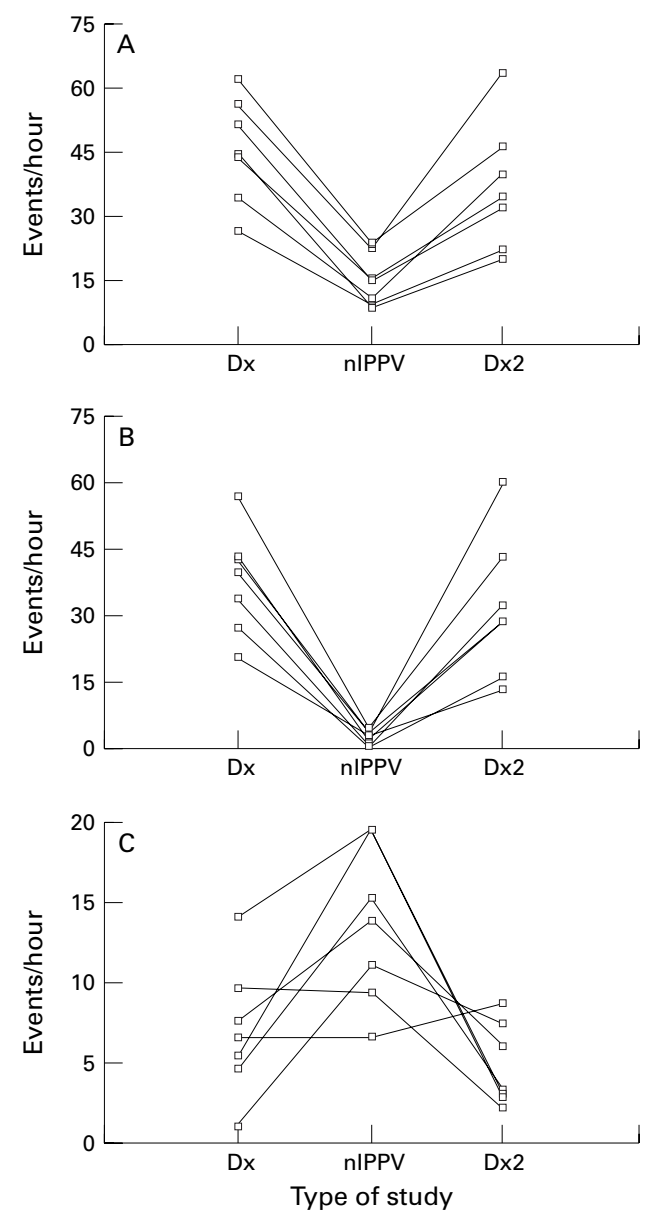

Figure 2 (A) Arousals; (B) arousals due to respiratory events; $(C)$ arousals due to non-respiratory events. $D x=$ baseline diagnostic; $n I P P V=$ on $n I P P V ; D \times 2=$ repeat study off $n I P P V$.

from three patients not reported due to technical difficulties). Arterial blood gas tensions were measured on awakening in six patients. There was little change in $\mathrm{pH}(7.41(0.02)$ in the afternoon versus $7.42(0.06)$ on awakening) or in $\mathrm{PaCO}_{2}(5.16(0.49) \mathrm{kPa}$ in the afternoon versus $4.96(0.35) \mathrm{kPa}$ on awakening).

ACCLIMATISATION TO NASAL IPPV

Most of the subjects adapted quickly to the ventilator and slept with the machine for a mean of 3.9 hours (range 1.5-6.75) on the first night. Ventilator settings used included a mean breath rate of $17 / \mathrm{min}$ (range 14-19), a mean tidal volume of 0.861 (range $0.75-1.00$ ), and a mean inspiratory time of $1.4 \mathrm{~s} \mathrm{(range} \mathrm{1.2-1.5).}$ These settings resulted in peak pressures in the range of 15-20 $\mathrm{cm} \mathrm{H}_{2} \mathrm{O}$ during effective ventilation in the absence of leaking. Two patients required $5 \mathrm{~cm} \mathrm{H}_{2} \mathrm{O}$ of PEEP to eliminate airway obstruction; no end expiratory pressure was used in the remaining nine patients.

Nasal IPPV was well tolerated by all patients whilst in hospital. All patients reported an improvement in their symptoms of excessive daytime sleepiness. A subjective improvement in exercise tolerance was reported in all but one patient. Complications of nIPPV were minor and included abdominal bloating in one patient, which was treated with the ingestion of 

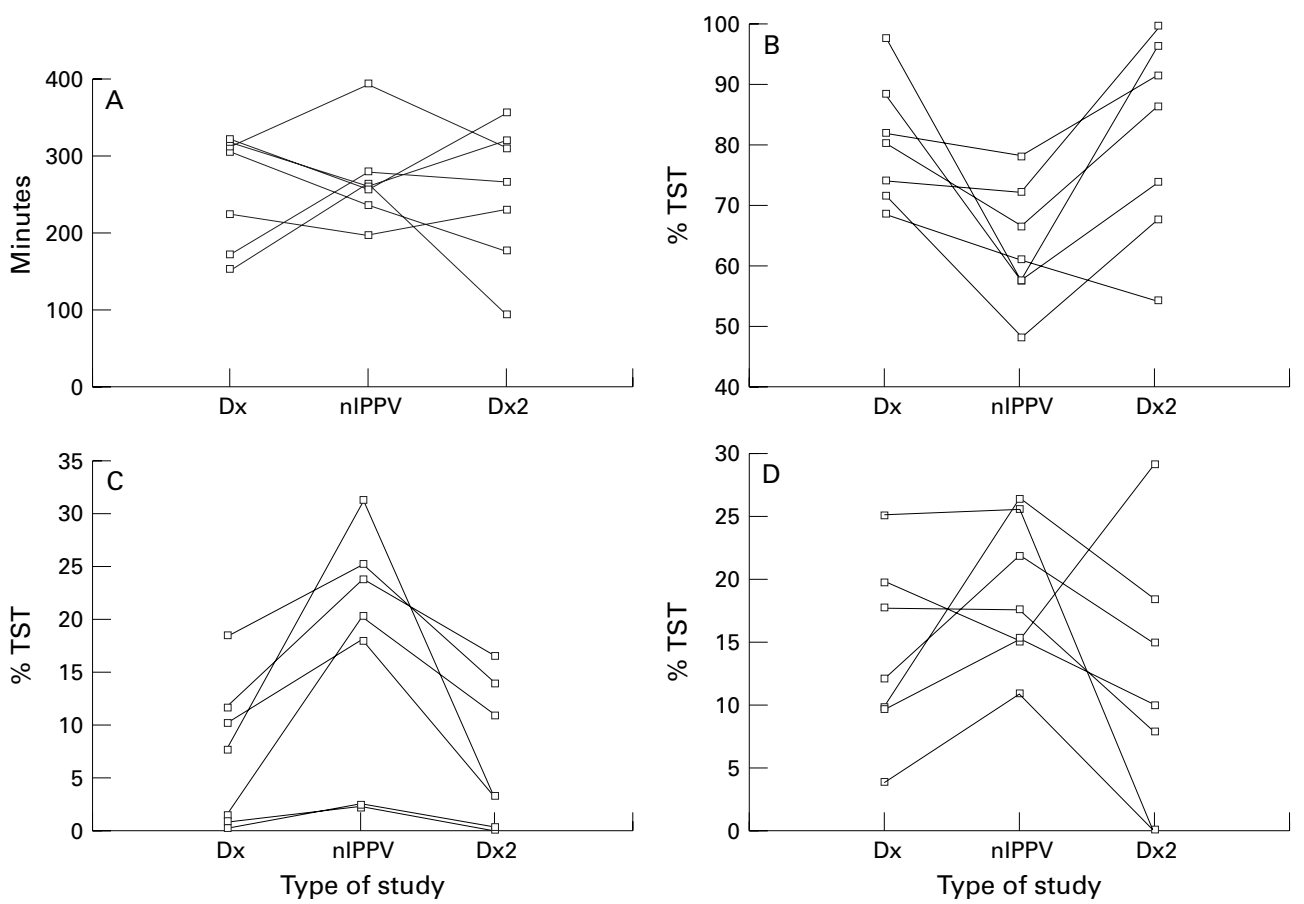

Figure 3 (A) Total sleep time; (B) stage 1/2 sleep; (C) slow wave sleep; (D) REM sleep. Dx = baseline diagnostic; $n I P P V=$ on $n I P P V ; D x 2=$ repeat study off $n I P P V$.

peppermint water before sleep. Three patients found the chinstrap uncomfortable. Patients required a period of 7.3 days (range $2-11$ days) to become completely comfortable sleeping with the machine and to be proficient in mask fitting and adjustment so as to undertake selfadministrated domiciliary care.

\section{DOMICILIARY EXPERIENCE}

Six of the 11 patients completed the three month trial. Three patients stopped using the device, one due to domestic difficulties, one due to cough associated with pneumonia, and one because of problems with sleep initiation. Two patients died during the course of the study, one at one month due to a pneumonia following a stroke and the second at one month from cardiac arrest while awake during the day, the likely cause being a primary cardiac arrhythmia. Neither patient had experienced a worsening of cardiac failure whilst using nIPPV. The patient who died following a cardiac arrest had been reviewed the previous day. At that time he was subjectively well with reports of increased exercise tolerance matched by an objective improvement in LVEF from $11 \%$ to $23 \%$. One patient required readmission

Table 2 Mean (SD) sleep study data

\begin{tabular}{|c|c|c|c|}
\hline & $\begin{array}{l}\text { Diagnostic } \\
\text { study }(n=7)\end{array}$ & $\begin{array}{l}n I P P V \\
(n=7)\end{array}$ & $\begin{array}{l}\text { Off nIPPV } \\
(n=7)\end{array}$ \\
\hline Total sleep time $(\mathrm{min})$ & $256(73)$ & $269(61)$ & $251(91)$ \\
\hline Sleep efficieny (\%) & $63(17)$ & $64(10)$ & $61(23)$ \\
\hline Stage $1 / 2(\%$ TST $)$ & $80(10)$ & $63(10) \dagger$ & $82(17)$ \\
\hline Slow wave sleep ( $\%$ TST) & $7(7)$ & $18(11) \dagger$ & $7(7)$ \\
\hline REM (\% TST) & $14(7)$ & $19(6)$ & $12(10)$ \\
\hline RDI (events/h) & $54(16)$ & $6(6)^{\star}$ & $53(20)$ \\
\hline Minimum $\mathrm{SaO}_{2}(\%)$ & $77(5)$ & $85(3) \dagger$ & $78(3)$ \\
\hline Arousal index (arousals $/ \mathrm{h}$ ) & $46(13)$ & $15(6)^{\star}$ & $37(15)$ \\
\hline Non-respiratory arousals (arousals $/ \mathrm{h}$ ) & $7(4)$ & $14(5)^{\star}$ & $5(3)$ \\
\hline
\end{tabular}

${ }^{\star} \mathrm{p}<0.01 ; \mathrm{tp}<0.05$ (two way ANOVA)

nIPPV $=$ nasal intermittent positive pressure ventilation; TST $=$ total sleep time, REM $=$ rapid eye movement sleep; $\mathrm{RDI}=$ respiratory disturbance index; $\mathrm{SaO}_{2}=$ oxyhaemoglobin saturation. during the course of the domiciliary trial because of worsening of heart failure. $\mathrm{He}$ responded well to a short term increase in his diuretic therapy and was discharged on his previous medications.

LEFT VENTRICULAR EJECTION FRACTION (LVEF)

LVEF increased from $25(9) \%$ to $31(10) \%$ (p $<0.05$ ) in the seven patients who continued to use the device for at least one month (five of these seven patients had used nIPPV for at least three months). Figure 4 shows the individual patient data and illustrates that the response to treatment was variable with only three patients showing an improvement in LVEF of $>6 \%$. Only one patient had a lower LVEF at the repeat study (from $27 \%$ to $24 \%$ ). In the three non-compliant patients only one had LVEF repeated which changed little (from $22 \%$ to $20 \%$ ). One patient died before a follow up measurement of LVEF was made.

\section{Discussion}

This study documents our experience with nIPPV in patients with chronic heart failure and central sleep apnoea. We have shown that CSR during sleep can be abolished with the use of nIPPV. Nasal ventilation leads to improved

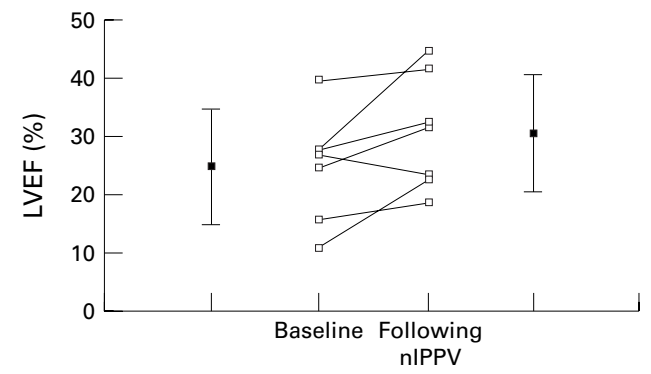

Figure 4 Change in LVEF with nIPPV. 
sleep architecture with a reduction in the arousal index, a decrease in stage $1 / 2$ sleep, and a significant increase in slow wave sleep. Although nasal ventilation was well tolerated by all patients whilst in hospital, compliance decreased markedly in the home environment. Nevertheless, those patients who continued to use the device on a regular basis reported an improvement in symptoms of daytime somnolence, fatigue, and breathlessness. Nasal IPPV was associated with an increase in LVEF in these patients.

Nasal IPPV is an effective treatment for patients with chronic respiratory failure due to neuromuscular disease and chest wall abnormalities. ${ }^{12}{ }^{13}$ The mechanism of improvement in these patients is thought to be due to reversal of nocturnal hypoventilation by mechanical ventilation. Many patients with hypercapnic respiratory failure exhibit nocturnal hypoventilation and central apnoea which is well controlled with nIPPV. This study demonstrates that the use of this modality of treatment can be extended to patients with central sleep apnoea due to congestive heart failure.

We hypothesised that nIPPV could be used to support ventilation during the apnoeic portion of the Cheyne-Stokes cycle, preventing alterations in blood gas tensions and thereby preventing arousal associated with these apnoeas. In fact, in many of our patients no spontaneous breathing effort was seen during ventilation, presumably because the arterial carbon dioxide tension was below the apnoeic threshold during sleep. This mechanism differs markedly from that of nCPAP which has been shown to decrease central respiratory events by increasing the arterial carbon dioxide tension, ${ }^{19}$ reducing the unstable respiratory control induced by hypocapnia. ${ }^{14}{ }^{15}$

The most significant finding of this study is the abolition of CSR during sleep using nIPPV. Reductions in RDI have been reported with the use of both supplemental oxygen and nCPAP. Hanley et $a l^{6}$ reported a reduction in the apnoea/hypopnoea index (AHI) from 30 (4.7) to 18.9 (2.4) events/h whilst breathing supplemental oxygen. Naughton et $a l^{8}$ found a decrease in AHI from 43.2 (4.9) to 14.7 (4.8) events/h on nCPAP following one month of domiciliary use. This finding is in contrast to the reports of Buckle et al, ${ }^{9}$ Guilleminault et $a l,{ }^{11}$ and Davies et $a l^{10}$ who showed no improvement in apnoea indices in shorter trials of CPAP. A number of explanations have been proposed for the conflicting results on CPAP including the prescription of inadequate pressures or the failure of an adequate acclimatisation period. The results of the present study show that effective treatment of severe CSR is possible following a short period of acclimatisation.

Residual respiratory events on nIPPV were due to obstructive events predominantly at sleep onset. The addition of PEEP at $5 \mathrm{~cm} \mathrm{H}_{2} \mathrm{O}$ in two patients decreased the number of these events, although intermittent mouth leaking makes the continuous maintenance of this PEEP almost impossible. These obstructive episodes are not surprising given that a number of authors have commented on the likely role of upper airway closure in the pathogenesis of central sleep apnoea. ${ }^{21}$

Sleep fragmentation has been reported as a dominant feature of sleep in patients with CSR and $\mathrm{CHF}$, with arousal usually occurring at the peak of the hyperpnoea. ${ }^{14}{ }^{15}$ Arousal indices were markedly reduced on nIPPV from 48.3 (13.8) to 13.8 (5.7) events/h. Indeed, the number of arousals on the nIPPV night was similar to that in normal subjects sleeping in our laboratory ${ }^{22}$ and below that found by other authors. ${ }^{23} \mathrm{~A}$ marked reduction in numbers of arousals occurred despite an increase in disturbance due to non-respiratory events, presumably from the device itself. A reduction in arousal index has also been reported with the use of oxygen (from 30 (4.7) to 13.8 (1.9) events $/ \mathrm{h}) .{ }^{6}$ Studies using nCPAP have reported conflicting results. Naughton $e t a l^{8}$ reported an improvement in one study (from 35.5 (5.5) to $23.5(4.4)$ events/h) whereas in two subsequent studies the improvement in arousal indices was non-significant. ${ }^{24} 25$ The reason for the improved effectiveness of nIPPV in the reduction of arousals is probably due to the greater control of CSR this treatment affords.

Abnormal sleep architecture with a reduction in the amount of slow wave sleep is a consistent finding in patients with CSR. Nasal IPPV resulted in an improvement in sleep architecture with a significant decrease in stage $1 / 2$ sleep and an increase in slow wave sleep. Both supplemental oxygen and nCPAP result in a reduction in stage $1 / 2$ sleep $^{68}$ but changes in slow wave sleep were not reported using oxygen and have only been reported in one study using nCPAP. ${ }^{25}$ We believe the increase in slow wave sleep on nIPPV may reflect the more effective control of apnoea, therefore permitting transition into deeper stages of sleep. There was a trend towards an increase in the amount of REM sleep during nIPPV, but this did not reach statistical significance. This may be due to the small number of patients and the fact that most of the patients had a low apnoea index in REM sleep during spontaneous breathing.

The sleep studies off nIPPV showed that chronic nIPPV does not appreciably change the underlying breathing disorder in patients with severe central sleep apnoea. The study off nIPPV confirms that nIPPV is responsible for the observed reduction in respiratory events and improvement in sleep quality. No significant difference was found between the initial and repeat diagnostic studies for any of the measured variables. The repeat diagnostic studies showed that the changes observed were due to nIPPV and not related to either night-to-night variability or acclimatisation to the laboratory.

We showed an improvement in LVEF in seven patients previously stable on medical treatment who continued to use nIPPV for at least one month. Naughton et $a l^{8}$ have shown improvement in LVEF following three months of nCPAP, with this result being confirmed in subsequent studies from the same group. ${ }^{24} 25$ The clinical significance of the observed 
improvement in LVEF in our study is difficult to determine in an uncontrolled study using such small numbers. The LVEF itself is a measure which is subject to a large amount of spontaneous variability in chronic heart failure. ${ }^{26}$ The present study only reports the results of patients compliant with nIPPV. A controlled study with a large number of subjects would be required to determine whether a significant improvement in cardiac function had occurred, and if this change was significant using an intention to treat analysis.

We believe the role of nIPPV will be to complement the existing therapies for CSR in heart failure. In patients who have an inadequate therapeutic response or fail to tolerate $\mathrm{nCPAP}$, nIPPV is a realistic alternative treatment modality. We believe that nasal ventilation is best applied by a highly trained therapist who can adjust machine settings to maintain patient comfort whilst achieving the desired ventilatory aims. In this study ventilation was commenced whilst the subject was an inpatient in hospital, allowing time an for intensive programme of education and acclimatisation. Four patients who had previously used nCPAP all commented on the increased comfort of nasal ventilation, with reports of improved sleep quality on nIPPV.

Long term domiciliary usage of the device was poor with only six of the 11 patients completing the three month trial. Three subjects stopped using nIPPV for the reasons outlined earlier. The two patients who died during the course of the study did so for reasons which were unrelated to their treatment. $\mathrm{CHF}$ is associated with a high risk of cardiac death (due to progressive heart failure or sudden death) and stroke. Five patients continue to use nasal ventilation long term and another has transferred to nCPAP. Two patients have continued treatment for more than two years and these patients are extremely reluctant to sleep without using nIPPV.

In summary, nasal ventilation can abolish Cheyne-Stokes respiration during sleep in patients with chronic heart failure. Abolition of CSR was associated with improvement in both sleep quality and symptoms of daytime somnolence, fatigue, and breathlessness. In those patients who continued to use the device at home, improvement in LVEF was documented. Further long term controlled studies are required to clarify the role of domiciliary therapy and to investigate the utility of nIPPV in comparison with existing therapies for central sleep apnoea during sleep.

The authors are grateful to the medical and nursing staff of the Centre for Respiratory Failure and Sleep Disorders, Roya Prince Alfred Hospital, for their practical assistance. We thank Michael Berthon-Jones and Stephen McNamara for helpful statistical advice. Supported by Medical Gases Australia and a project grant from the National Health and Medical Research Council of Australia.
1 Javaheri S, Parker TJ, Wexler L, et al. Occult sleepdisordered breathing in stable congestive heart failure. Ann disordered breathing in stable

2 Dowdell WT, Javaheri S, McGinnis W. Cheyne-Stokes respiration presenting as sleep apnea syndrome. Clinical and polysomnographic features. Am Rev Respir Dis 1990;141:871-9.

3 Hanly PJ, Millar TW, Steljes DG, et al. Respiration and abnormal sleep in patients with congestive heart failure. Chest 1989;96:480-8.

4 Findley LJ, Zwillich CW, Ancoli Israel S, et al. CheyneStokes breathing during sleep in patients with left ventricular heart failure. South Med $\mathcal{f}$ 1985;78:11-5.

5 Hanly PJ, Zuberi-Khokhar NS. Increased mortality associated with Cheyne-Stokes respiration in patients with congestive heart failure. Am 7 Respir Crit Care Med 1996;153:272-6.

6 Hanly PJ, Millar TW, Steljes DG, et al. The effect of oxygen on respiration and sleep in patients with congestive heart failure. Ann Intern Med 1989;111:777-82.

7 Javaheri S, Parker TJ, Wexler L, et al. Effect of theophylline on sleep-disordered breathing in heart failure. $N$ Engl $\mathcal{F}$ Med 1996;335:562-7.

8 Naughton MT, Liu PP, Bernard DC, et al. Treatment of congestive heart failure and Cheyne-Stokes respiration during sleep by continuous positive airway pressure. Am $\mathcal{F}$ Respir Crit Care Med 1995;151:92-7.

9 Buckle P, Millar T, Kryger M. The effect of short-term nasal CPAP on Cheyne-Stokes respiration in congestive heart failure. Chest 1992;102:31-5.

10 Davies RJ, Harrington KJ, Ormerod OJ, et al. Nasal continuous positive airway pressure in chronic heart failure with sleep-disordered breathing. Am Rev Respir Dis with sleep-disor

11 Guilleminault C, Clerk A, Labanowski M, et al. Cardiac failure and benzodiazepines. Sleep 1993;16:524-8.

12 Ellis ER, Bye PTP, Bruderer JW, et al. Treatment of respiratory failure during sleep in patients with neuromuscular disease. Am Rev Respir Dis 1987;135:148-52.

13 Ellis ER, Grunstein RR, Chan S, et al. Noninvasive ventilatory support during sleep improves respiratory failure in kyphoscoliosis. Chest 1988;94:811-5.

14 Naughton M, Benard D, Tam A, et al. Role of hyperventilation in the pathogenesis of central sleep apneas in patients 330-8.

15 Hanly P, Zuberi N, Gray R. Pathogenesis of Cheyne-Stokes respiration in patients with congestive heart failure Relationship to arterial $\mathrm{PCO}_{2}$. Chest 1993;104:1079-84.

16 Rechtschaffen A, Kales A. A manual of standardized terminology, techniques and scoring system for sleep stages of human subjects. Los Angeles: University of California at Los Angeles, 1968.

17 American Sleep Disorders Association Task Force. EEG arousals: scoring rules and examples. Sleep 1992;15:175-

18 Grunstein RR, Ho KY, Berthon Jones M, et al. Central sleep apnea is associated with increased ventilatory response to carbon dioxide and hypersecretion of growth hormone in patients with acromegaly. Am F Respir Crit Care Med 1994; 150:496-502.

19 Naughton MT, Benard DC, Rutherford R, et al. Effect of continuous positive airway pressure on central sleep apnea and nocturnal $\mathrm{PCO}_{2}$ in heart failure. Am 7 Respir Crit Care Med 1994;150:1598-604

20 Badr MS, Toiber F, Skatrud JB, et al. Pharyngeal narrowing/ occlusion during central sleep apnea. $\mathcal{f}$ Appl Physiol 1995; 78:1806-15.

21 Alex CG, Onal E, Lopata M. Upper airway occlusion during sleep in patients with Cheyne-Stokes respiration. Am Rev Respir Dis 1986;133:42-5.

22 Lawrence S, Edwards N, Grunstein R, et al. Frequency of arousals in sleep laboratory control subjects (abstract). 1995 Annual Scientific Meeting, Australasian Sleep Association: $\mathrm{O} 7$.

23 Mathur R, Douglas NJ. Frequency of EEG arousals from nocturnal sleep in normal subjects. Sleep 1995;18:330-3.

24 Naughton MT, Benard DC, Liu PP, et al. Effects of nasal CPAP on sympathetic activity in patients with heart failure and central sleep apnea. Am 7 Respir Crit Care Med and central sleep

25 Granton JT, Naughton MT, Benard DC, et al. CPAP improves inspiratory muscle strength in patients with heart failure and central sleep apnea. Am $\mathcal{F}$ Respir Crit Care Med 1996;153:277-82.

26 Narahara KA. Spontaneous variability of ventricular function in patients with chronic heart failure. The Western Enoximone Study Group and the REFLECT investigators. Am f Med 1993;95:513-8. 